\title{
Intratumoral and peritumoral radiomics for the pretreatment prediction of pathological complete response to neoadjuvant chemotherapy based on breast DCE-MRI
}

Nathaniel M. Braman ${ }^{1 *}$, Maryam Etesami ${ }^{2}$, Prateek Prasanna ${ }^{1}$, Christina Dubchuk ${ }^{2}$, Hannah Gilmore ${ }^{2}$, Pallavi Tiwari ${ }^{1}$, Donna Plecha ${ }^{2}$ and Anant Madabhushi ${ }^{1}$

\begin{abstract}
Background: In this study, we evaluated the ability of radiomic textural analysis of intratumoral and peritumoral regions on pretreatment breast cancer dynamic contrast-enhanced magnetic resonance imaging (DCE-MRI) to predict pathological complete response (pCR) to neoadjuvant chemotherapy (NAC).

Methods: A total of 117 patients who had received NAC were retrospectively analyzed. Within the intratumoral and peritumoral regions of T1-weighted contrast-enhanced MRI scans, a total of 99 radiomic textural features were computed at multiple phases. Feature selection was used to identify a set of top pCR-associated features from within a training set $(n=78)$, which were then used to train multiple machine learning classifiers to predict the likelihood of pCR for a given patient. Classifiers were then independently tested on 39 patients. Experiments were repeated separately among hormone receptor-positive and human epidermal growth factor receptor 2-negative $\left(\mathrm{HR}^{+}, \mathrm{HER} 2^{-}\right)$and triple-negative or HER2 ${ }^{+}$(TN/HER2 ${ }^{+}$) tumors via threefold cross-validation to determine whether receptor status-specific analysis could improve classification performance.

Results: Among all patients, a combined intratumoral and peritumoral radiomic feature set yielded a maximum $A \cup C$ of $0.78 \pm 0.030$ within the training set and 0.74 within the independent testing set using a diagonal linear discriminant analysis (DLDA) classifier. Receptor status-specific feature discovery and classification enabled improved prediction of $\mathrm{pCR}$, yielding maximum AUCs of $0.83 \pm 0.025$ within the $\mathrm{HR}^{+}, \mathrm{HER}^{-}$group using DLDA and $0.93 \pm 0.018$ within the TN/HER2 ${ }^{+}$group using a naive Bayes classifier. In HR ${ }^{+}$, HER2 ${ }^{-}$breast cancers, non-pCR was characterized by elevated peritumoral heterogeneity during initial contrast enhancement. However, TN/HER2 ${ }^{+}$tumors were best characterized by a speckled enhancement pattern within the peritumoral region of nonresponders. Radiomic features were found to strongly predict PCR independent of choice of classifier, suggesting their robustness as response predictors.

Conclusions: Through a combined intratumoral and peritumoral radiomics approach, we could successfully predict $\mathrm{PCR}$ to NAC from pretreatment breast DCE-MRI, both with and without a priori knowledge of receptor status. Further, our findings suggest that the radiomic features most predictive of response vary across different receptor subtypes.
\end{abstract}

Keywords: Imaging, MRI, Neoadjuvant chemotherapy, Treatment response, Radiomics, Personalized medicine

\footnotetext{
* Correspondence: nathaniel.braman@case.edu

'Department of Biomedical Engineering, Case Western Reserve University,

Cleveland, OH 44106, USA

Full list of author information is available at the end of the article
} 


\section{Background}

For the $10 \%$ to $20 \%$ of the 230,000 patients diagnosed with breast cancer each year [1] who have locally advanced breast cancer, it is imperative to receive effective treatment as quickly as possible. These patients sit at a critical clinical juncture: Their tumor has spread beyond the breast to the chest wall, skin, or lymph nodes but has not yet metastasized further. Even with treatment, most patients with locally advanced breast cancer will develop distant metastases [2]. Neoadjuvant chemotherapy (NAC) is often a first line of defense in the treatment of locally advanced breast cancer. Administered prior to surgery, NAC can reduce tumor extent, increase a patient's surgical options, and reduce metastasis [3]. The ideal outcome of NAC is the complete absence of residual invasive tumor cells within excised breast tissue following NAC, or pathological complete response (pCR), which strongly predicts favorable prognosis as compared with patients who experience partial or no response (non-pCR) $[4,5]$. Less than 10$50 \%$ of breast cancer patients who undergo NAC achieve pCR, depending on receptor status subtype [6], and thus there is a need for reliable noninvasive pretreatment predictors of pCR that can enable better targeting of NAC and prevent a delay in effective treatment for patients with nonresponding or progressive tumors.

Because of its high sensitivity to tumor presence and angiogenic changes, dynamic contrast-enhanced magnetic resonance imaging (DCE-MRI) is the preferred imaging modality in the NAC setting and has been demonstrated to effectively predict pCR following an early treatment period. For instance, changes in volumetric and kinetic parameters perform well in $\mathrm{pCR}$ prediction but afford no intuition with regard to $\mathrm{PCR}$ prior to treatment [7-10]. There remains a shortage of reliable clinical pCR indicators based on DCE-MRI that do not require previous NAC administration $[7,10]$.

Radiomic textural analysis-the high-throughput extraction of quantitative imaging features characterizing the spatial relationships and consistency of signal intensities-within the tumor region has been shown to enable pCR prediction across a range of cancer types and imaging modalities [11]. Many of these features, such as Haralick gray-level co-occurrence matrix-based features, quantify enhancement heterogeneity, which has been shown to predict aggressive growth, unfavorable prognosis, and poor treatment response in breast cancer [12-19]. Within the realm of breast cancer, DCE-MRI radiomics has been shown to be effective in predicting breast cancer biology, including receptor status [15, 20, 21], subtype [13, 20-23], and genomics [12, 24, 25].

Previous work exploring radiomics within the whole breast parenchyma has suggested the importance of studying the tumor's physiological environment in building predictive models of tumor response and outcome
[22, 26-28]. However, evidence suggests that the peritumoral region - the area immediately surrounding the tumor mass-may possess valuable outcome-related information that is not effectively captured by radiomic analysis of the bulk parenchyma. Angiogenic [29] and lymphangiogenic [30] activity, as well as peritumoral invasion of lymphatics and blood vessels [31, 32], is a predictor of survival that manifests within the peritumoral region on imaging studies. Signatures of immune response present within peripheral breast tissue, such as stromal response [33] and peritumoral lymphocytic infiltration [34], also have documented correlations with outcome. Therefore, intratumoral and parenchymal radiomics may be missing crucial markers of response located in the surrounding tumor microenvironment.

In this study, we explored the application of intratumoral and peritumoral radiomics for the noninvasive prediction of pCR in NAC recipients based on pretreatment DCE-MRI. We identified a radiomic profile of response consisting of the intratumoral and peritumoral features that best collectively distinguishes pCR and non-pCR. We assessed the added value of peritumoral radiomics and used our radiomic profile to predict $\mathrm{pCR}$. In the second half of this study, we divided our dataset by receptor type and again identified radiomic profiles of response specific to receptor subgroup. We then investigated whether a subtype-specific profile of intratumoral and peritumoral radiomics enhances the ability to predict response.

\section{Methods}

This Health Insurance Portability and Accountability Act of 1996 ("HIPAA") regulations-compliant study was approved by institutional review board at the University Hospitals Case Medical Center, and the need for informed consent was waived. Figure 1 depicts patient selection and overall experimental design. All 118 patients with biopsy-proven primary breast cancer who received NAC prior to breast surgery and had breast DCE-MRI before initiation of NAC at the Cleveland University Hospitals network between 1 March 2012 and 15 May 2016 were retrospectively included in this study. One patient was eliminated from the dataset for insufficient tumor volume on imaging, leaving a dataset of 117 patients (Table 1). Human epidermal growth factor receptor 2-negative (HER2 ${ }^{-}$) patients received doxorubicin and cyclophosphamide every 2 weeks for four cycles followed by four cycles of paclitaxel. All $\mathrm{HER}^{+}$patients received treatment with docetaxel and trastuzumab every 3 weeks for six cycles. The HER $2^{+}$ treatment regimen was also supplemented with pertuzumab and/or carboplatin on a patient-by-patient basis, depending on disease severity and availability at the time of treatment.

Breast tissue was processed for histopathological analysis according to standard anatomical pathology protocols in compliance with all College of American Pathologists standards. Tissues were fixed in $10 \%$ neutral buffered 


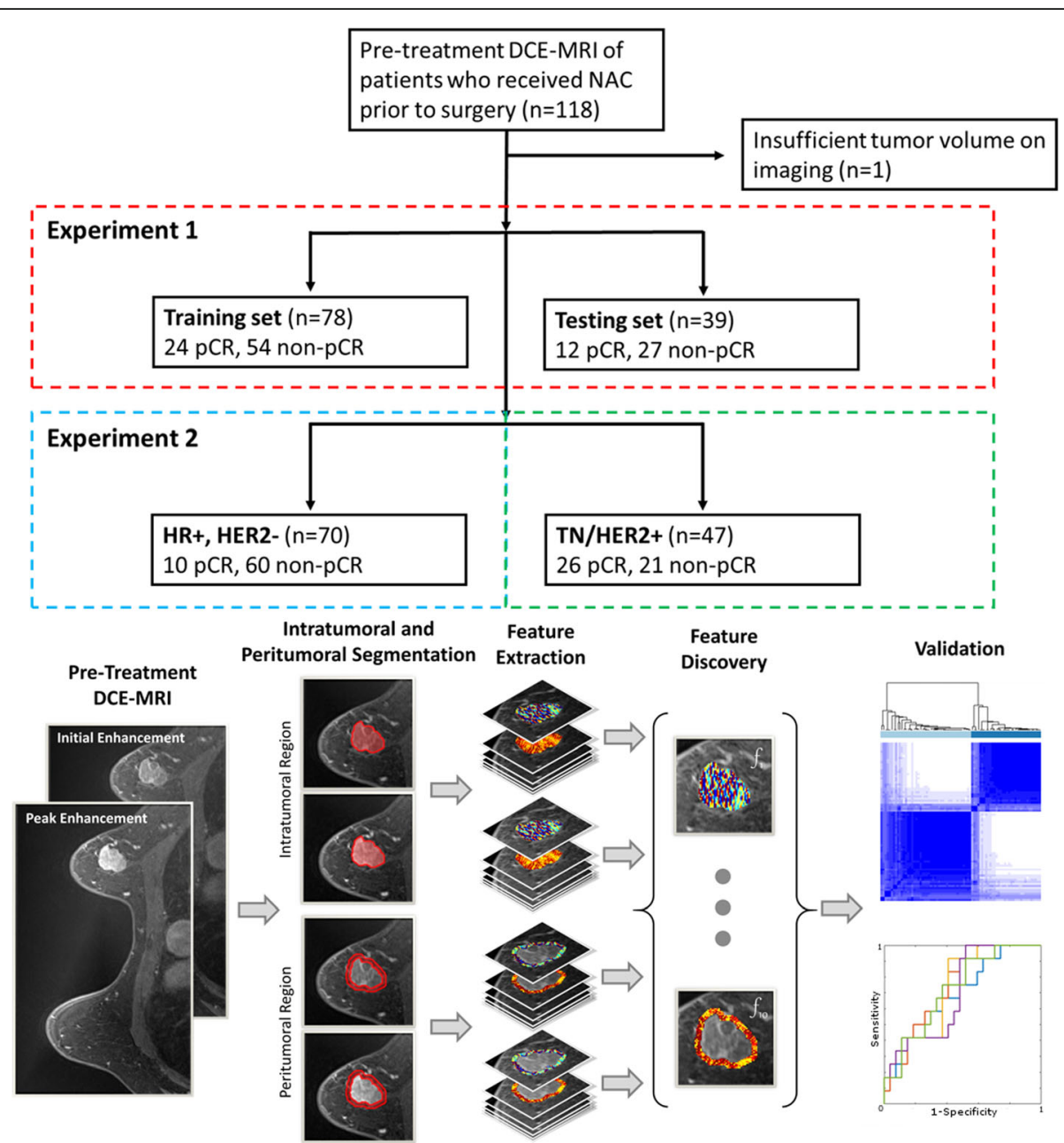

Fig. 1 Top: Patient selection flowchart for experiments 1-3. Bottom: Radiomic pathological complete response (pCR) prediction pipeline. DCE-MRI Dynamic contrast-enhanced magnetic resonance imaging, ER Estrogen receptor, HER2 Human epidermal growth factor receptor 2, HR Hormone receptor, NAC Neoadjuvant chemotherapy, TN Triple-negative

formalin and processed overnight in standard tissue processors. Slides were cut at $5 \mu \mathrm{m}$ and stained on a Ventana automated staining system (Ventana Medical Systems, Tucson, AZ, USA). All pathological diagnoses were rendered by the breast pathology subspecialty service at University Hospitals Cleveland Medical Center (Cleveland, OH, USA). Pathological response to NAC was defined as an absence of invasive cancer in the breast surgical specimen (ypT0/is). Following the course of NAC, 36 patients achieved $\mathrm{pCR}$ in the breast as observed on the surgical specimen. Of these 36 patients who had a complete response in the breast (defined at T0 or Tis), 29 also were lymph node-negative at the time of surgery. Of the remaining seven patients with positive lymph nodes, all but one had only scant disease remaining in the lymph nodes following NAC (three were ypN1mi, three were ypN1a, and one was ypN2a). The remaining 81 patients had partial or no response to NAC in the breast (non-pCR).
Patients considered in this study had been scanned previously at different sites within the Cleveland University Hospitals network and on different scanner platforms with different magnets at both $1.5 \mathrm{~T}$ (MAGNETOM Avanto [Siemens Healthcare, Erlangen, Germany], MAGNETOM Espree [Siemens Healthcare], and Intera [Philips Medical Systems, Best, The Netherlands]) and 3 T (MAGNETOM Verio [Siemens Healthcare], Ingenia [Philips Medical Systems], and Ingenuity [Philips Medical Systems]). Axial fast low angle shot fat-saturated T1-weighted 3D scans were obtained before and after contrast agent injection. Pixel sizes ranged from $0.50 \times 0.50 \mathrm{~mm}$ to $1.0 \times$ $1.0 \mathrm{~mm}$, with an average of $0.76 \times 0.76 \mathrm{~mm}$. Slice thickness ranged from 0.9 to $3 \mathrm{~mm}$ (average $1.3 \mathrm{~mm}$ ).

An initial fat-saturated T1-weighted precontrast scan was first collected. Then $0.1 \mathrm{mmol} / \mathrm{kg}$ of either OptiMARK (Mallinckrodt, St. Louis, MO, USA) or MultiHance (Bracco Diagnostics, Monroe Township, NJ, USA) gadolinium- 
Table 1 Breakdown of dataset by pathological complete response status

\begin{tabular}{|c|c|c|}
\hline & $\mathrm{pCR}$ & Non-pCR \\
\hline Number of patients & 36 & 81 \\
\hline Mean age, years & $53(23-72)$ & $48(26-79)$ \\
\hline \multicolumn{3}{|l|}{ Receptor status } \\
\hline $\mathrm{ER}^{+}$ & 17 & 66 \\
\hline $\mathrm{PR}^{+}$ & 15 & 58 \\
\hline HER2 ${ }^{+}$ & 16 & 12 \\
\hline $\mathrm{TN}$ & 10 & 9 \\
\hline 1.5-T DCE-MRI & 30 & 60 \\
\hline Scanner 1 & 17 & 40 \\
\hline Scanner 2 & 12 & 15 \\
\hline Scanner 3 & 1 & 5 \\
\hline 3-T DCE-MRI & 6 & 21 \\
\hline Scanner 4 & 0 & 2 \\
\hline Scanner 5 & 3 & 10 \\
\hline Scanner 6 & 3 & 9 \\
\hline \multicolumn{3}{|l|}{ Lesion diameter, cm } \\
\hline Mean & 3.9 & 4.2 \\
\hline SD & 2.4 & 2.6 \\
\hline \multicolumn{3}{|l|}{ Stage } \\
\hline I & 5 & 6 \\
\hline$\|$ & 18 & 43 \\
\hline III & 13 & 31 \\
\hline IV & 0 & 1 \\
\hline Enhancing mass & 29 & 69 \\
\hline Non-mass enhancement & 7 & 12 \\
\hline
\end{tabular}

Abbreviations: DCE-MRI Dynamic contrast-enhanced magnetic resonance imaging, ER Estrogen receptor, HER2 Human epidermal growth factor receptor $2, p C R$ Pathological complete response, $P R$ Progesterone receptor, TN Triple-negative

based contrast agent was injected intravenously. A first postcontrast scan was collected 2 minutes after contrast agent injection. Four subsequent postcontrast images were acquired at intervals of 90 seconds, resulting in five postcontrast images for each patient $(t=2,3.5,5$, 6.5 , and 8 minutes).

Two breast radiologists with 23 years (DP) and 3 years (ME) of experience reviewed and annotated lesion boundaries as the intratumoral region in consensus. A 2.5- to 5-mm (depending on pixel size) radius surrounding the tumor was defined as the peritumoral region. Lesion annotation and peritumoral mask generation are described in full in Additional file 1: Supplementary Methods.

\section{Feature extraction}

All feature extraction was performed using software previously developed in the Center for Computational Imaging and Personalized Diagnostics, Case Western
Reserve University, implemented on a MATLAB release 2016a platform (MathWorks, Natick, MA, USA). A total of 99 textural descriptors were computed voxelwise across all slices for each phase, and location was analyzed. A list of textural features explored, along with descriptions and biological rationales, is provided in Table 2.

Physiologically based DCE-MRI pharmacokinetic (PK) parameters (rate of contrast agent uptake into tumor from blood $\left[K^{\text {Trans }}\right]$, rate of contrast agent transport from tumor to blood $\left[K_{\mathrm{ep}}\right]$, and tumor volume $\left[\mathrm{V}_{\mathrm{e}}\right]$ ) were also computed from voxel intensities across each postcontrast phase using the Tofts model [35] for comparison against intratumoral and peritumoral radiomics.

First-order statistics (mean, median, SD, skewness, and kurtosis) of descriptor values were calculated for each location and phase-analyzed, resulting in 495 statistical features for each location and phase analyzed. A total of 1980 features for each scan were obtained, with 990 statistical features each extracted from the intratumoral and peritumoral regions. Feature values were normalized between -1 and 1 .

All quantitative data analysis was implemented using MATLAB release 2016a software unless otherwise stated.

\section{Experiment 1: pCR prediction among all-comers}

Groups of $24 \mathrm{pCR}$ and 54 non-pCR patients were randomly sorted into a 78-patient training set. The remaining 39 patients, including $12 \mathrm{pCR}$ and 27 non-pCR, were held out as the independent testing set. Training and testing set information is included in Table 3.

\section{Feature discovery}

Minimum redundancy, maximum relevancy (mRMR) feature selection [36] was implemented to select the top 10 pCR-associated features across 200 iterations of threefold cross-validation within the training cohort. mRMR was used to identify a set of features that maximally distinguished two classes ( $\mathrm{pCR}$ and non-pCR) while minimizing intrafeature correlation. Features were ranked by frequency of selection, and the bottom $90 \%$ of features were eliminated. Feature selection was repeated within the reduced feature set, and features were again ranked by selection frequency. Redundant overlapping features were eliminated from this list to yield a final set of top pCRassociated radiomic features. The number of features selected was capped at ten to prevent overfitting due to the "curse of dimensionality" [37] arising from an overabundance of features relative to sample size. mRMR was performed using MATLAB software with the Feature Selection Toolbox for C (FEAST; Czech Academy of Sciences, Prague, Czech Republic) [38]. 
Table 2 Radiomic feature families extracted from the intratumoral and peritumoral regions

\begin{tabular}{|c|c|c|c|}
\hline Feature group & Quantity & Description & Rationale \\
\hline Laws energy measures & 25 & $\begin{array}{l}\text { Response to } 5 \text {-pixel } \times 5 \text {-pixel filter targeting } \\
\text { combination of specific textural enhancement } \\
\text { patterns in the } x \text { and } y \text { directions. Descriptors } \\
\text { include all combinations of five } 1 D \text { filters: } \\
\text { level }(L) \text {, edge }(E) \text {, spot }(S) \text {, wave }(W) \text {, and } \\
\text { ripple (R). }\end{array}$ & $\begin{array}{l}\text { May possibly detect patterns of heterogeneous } \\
\text { enhancement and abnormal structure; have } \\
\text { previously been shown to enable quantification } \\
\text { of TILs by lung CT [57]. }\end{array}$ \\
\hline Gabor features & 48 & $\begin{array}{l}\text { Detection of edges through response to Gabor } \\
\text { wavelet features. Each descriptor quantifies } \\
\text { response to a given Gabor filter at a specific } \\
\text { frequency ( } f=0,2,4,8,16 \text {, or } 32 \text { ) and orientation } \\
(\theta=0 \text { degrees, } 22.5 \text { degrees, } 45 \text { degrees, } 67.5 \\
\text { degrees, } 90 \text { degrees, } 112.5 \text { degrees, } 135 \text { degrees, } \\
167.5 \text { degrees). }\end{array}$ & $\begin{array}{l}\text { May possibly capture changes in tumor } \\
\text { microarchitecture on account of glandular } \\
\text { morphology or detect the presence of } \\
\text { TILs [57]. TILs have been shown to be } \\
\text { prognostic of better survival and NAC } \\
\text { response [54]. }\end{array}$ \\
\hline Haralick features & 13 & $\begin{array}{l}\text { Quantify heterogeneity and entropy of local } \\
\text { intensity texture as represented by the } \\
\text { gray-level co-occurrence matrix within a } \\
\text { 5-pixel } \times 5 \text {-pixel window. }\end{array}$ & $\begin{array}{l}\text { Regional changes in Haralick features following } \\
\text { treatment have been shown to predict pCR in } \\
\text { breast cancer [19]. }\end{array}$ \\
\hline $\begin{array}{l}\text { Co-occurrence of Local } \\
\text { Anisotropic Gradient } \\
\text { Orientations (CoLIAGe) } \\
\text { features }\end{array}$ & 13 & $\begin{array}{l}\text { Apply Haralick metrics to dominant intensity } \\
\text { gradient orientations within a } 5 \text {-pixel } \times 5 \text {-pixel } \\
\text { window, quantifying patterns of local gradient } \\
\text { alignment }[59,60] \text {. Some descriptors quantify } \\
\text { homogeneity of gradient orientations (e.g., } \\
\text { CoLIAGe information measure of correlation), } \\
\text { whereas others compute their disorder (e.g., } \\
\text { CoLIAGe entropy). }\end{array}$ & $\begin{array}{l}\text { CoLIAGe entropy has previously been demonstrated } \\
\text { to be effective in distinguishing breast cancer } \\
\text { subtypes }[59,60] \text {. }\end{array}$ \\
\hline
\end{tabular}

Abbreviations: CT Computed tomography, NAC Neoadjuvant chemotherapy, $p C R$ Pathological complete response, TIL Tumor-infiltrating lymphocyte

This top radiomic feature set was analyzed using box-and-whisker plots and qualitative feature maps comparing feature expression between representative $\mathrm{pCR}$ and non-pCR tumors. Box plots for the top feature identified were compared against the performance of PK parameters that are physiological measures of tumor contrast uptake. Hematoxylin and eosin staining of pretreatment diagnostic core biopsy specimens for representative patients was also qualitatively examined by a breast pathologist (HG) with 10 years of experience to explore and potentially identify a possible physiological basis for the top-expressing radiomic features.

\section{Validation}

Two distinct strategies were used to validate our intratumoral and peritumoral radiomic features set. First, to validate the importance of a combined intratumoral and peritumoral radiomic approach in identifying $\mathrm{pCR}$ in a biologically heterogeneous dataset, consensus clustering [39] of the top ten intratumoral and peritumoral radiomic features was performed within the training group using the ConsensusClusterPlus package [40] in $\mathrm{R}$ [41]. One thousand iterations of hierarchical consensus clustering $(k=2)$ by Pearson distance [42] were performed with $80 \%$ random patient resampling between runs. Clustering results were visualized in a consensus cluster

Table 3 Breakdown of dataset by experiment

\begin{tabular}{|c|c|c|c|c|}
\hline & Experiment 1: training & Experiment 1: testing & Experiment 2: $\mathrm{HR}^{+}, \mathrm{HER}^{-}$ & Experiment 3: TN/HER2 ${ }^{+}$ \\
\hline Number of patients & 78 & 39 & 70 & 47 \\
\hline \multicolumn{5}{|c|}{ Type of pathological response } \\
\hline $\mathrm{pCR}$ & 24 & 12 & 10 & 26 \\
\hline Non-pCR & 54 & 27 & 60 & 21 \\
\hline \multicolumn{5}{|l|}{ Receptor group } \\
\hline $\mathrm{HR}^{+}, \mathrm{HER}^{-}$ & 46 & 24 & 70 & 0 \\
\hline $\mathrm{TN}^{\mathrm{H}} \mathrm{HER} 2^{+}$ & 32 & 15 & 0 & 47 \\
\hline \multicolumn{5}{|l|}{ Scanner strength } \\
\hline $1.5 \mathrm{~T}$ & 63 & 27 & 54 & 36 \\
\hline $3 T$ & 15 & 12 & 16 & 11 \\
\hline
\end{tabular}

Abbreviations: HER2 Human epidermal growth factor receptor 2, HR Hormone receptor, $p C R$ Pathological complete response, TN Triple-negative 
heatmap where shading indicated the consensus between patients or the frequency at which a pair of patients was clustered together. Clustering results were compared against pCR ground truth to assess the ability of top features to identify responders without a priori knowledge of patient biology or outcome. Feature discovery and consensus clustering were repeated in the subsets of intratumoral and peritumoral features only and compared against a combined clustering of intratumoral and peritumoral radiomic features. Consensus clustering was also performed and compared using all 15 PK statistical features from the entire dataset.

Next, we assessed the ability of an intratumoral and peritumoral radiomic feature set to predict $\mathrm{pCR}$ among new patients. The ability of top features to predict $\mathrm{pCR}$ was validated using five different machine learning classifiers to verify that successful pCR prediction was driven by our features as opposed to the choice of classifier. The following classifiers [43] were explored: linear discriminant analysis (LDA), diagonal linear discriminant analysis (DLDA), quadratic discriminant analysis, naive Bayes, and support vector machine. Within the training group, classifiers were iteratively trained and applied across 50 iterations of threefold cross-validation to first assess pCR prediction within the training set. Classifiers were then trained on the training set and tested by prediction of response within the independent testing set. Training and classification of each classifier was repeated ten times to sequentially include the top ten radiomic features. Performance was assessed by AUC, accuracy, sensitivity (ability to correctly identify patients who achieved pCR), and specificity (ability to correctly identify patients who did not achieve pCR).

\section{Experiment 2: pCR prediction with separation by receptor type}

Table 3 lists receptor status groups evaluated in experiment 2. The subset of 70 patients (10 pCR, 60 non-pCR) who were estrogen receptor (ER)- or progesterone receptor (PR)-positive, as well as HER2 ${ }^{-}$, were grouped into a hormone receptor (HR)-positive, HER2 ${ }^{-}\left(\mathrm{HR}^{+}, \mathrm{HER} 2^{-}\right)$cohort. The remaining 47 patients included 19 triple-negative (TN) and $28 \mathrm{HER}^{+}$tumors, and these patients were analyzed as a combined 47-patient TN/HER2 $2^{+}$cohort $(26 \mathrm{pCR}$ and 21 non-pCR). Owing to a low response rate within the $\mathrm{HR}^{+}$, HER2 ${ }^{-}$group and fewer total samples within the TN/ $\mathrm{HER}^{+}$group, neither was divided into training and testing groups. Instead, for experiment 2 alone, threefold cross-validation was used for classifier training and testing.

\section{Feature discovery}

Feature discovery was repeated among the entire subset of patients with $\mathrm{HR}^{+}, \mathrm{HER} 2^{-}$breast cancer. Because only
10 of the $70 \mathrm{HR}^{+}, \mathrm{HER}^{-}$patients experienced pCR, non$\mathrm{pCR}$ tumors were randomly downsampled to a group of 20 for each iteration of mRMR cross-validation to reduce selection of feature sets biased toward non-pCR samples. Feature discovery was performed within the group of TN/ HER $^{+}$patients without downsampling. Features were evaluated using box plots and qualitative feature maps.

\section{Validation}

Classifiers were trained in 50 iterations of threefold cross-validation using the top 1 through 10 features identified within each receptor status group. $\mathrm{PCR}$ prediction was assessed by mean AUC, accuracy, sensitivity, and specificity across cross-validation repetitions.

\section{Results}

\section{Experiment 1: pCR prediction among all-comers Feature discovery}

The top ten radiomic feature set obtained during feature discovery within the training set are listed in Table 3. CoLlAGe features are most frequently represented, occupying six of the top features. The remaining four members are Laws features. Intratumoral features and features extracted from the initial postcontrast phase are both more prevalent within the top feature set, each comprising six top features. Additionally, the top two features, CoLlAGe information measure of correlation 1 and Laws spot-ripple, were both expressed intratumorally during the first postcontrast phase.

pCR was most frequently characterized by increased intratumoral kurtosis of CoLlAGe information measure of correlation 1 during the initial enhancement phase, a feature quantifying homogeneity of intensity gradient orientations. In Fig. 3a, increased pCR expression of CoLlAGe information measure of correlation 1 within the intratumoral region during the initial postcontrast phase is shown in representative feature maps. The histology of representative patients revealed a high presence of tumorinfiltrating lymphocytes (TILs) in pCR, as well as sclerosis and necrosis in non-pCR biopsy samples (Fig. 3d). Box plots show elevated expression among patients who experienced $\mathrm{pCR}$ as compared with non-pCR in both the training and testing sets (Additional file 2). Features 2 and 3-skewness of intratumoral initial Laws spot-ripple and SD of peritumoral peak Laws level-spot-showed that $\mathrm{pCR}$ and non-pCR differed in patterns of enhancement texture at both phases and locations. The fourth top feature-kurtosis of CoLlAGe sum entropy-was expressed more strongly within the peritumoral region of nonresponders during the initial enhancement phase.

\section{Validation}

In Fig. 2a, the combined intratumoral and peritumoral feature set yields distinct pCR-associated clusters, with 


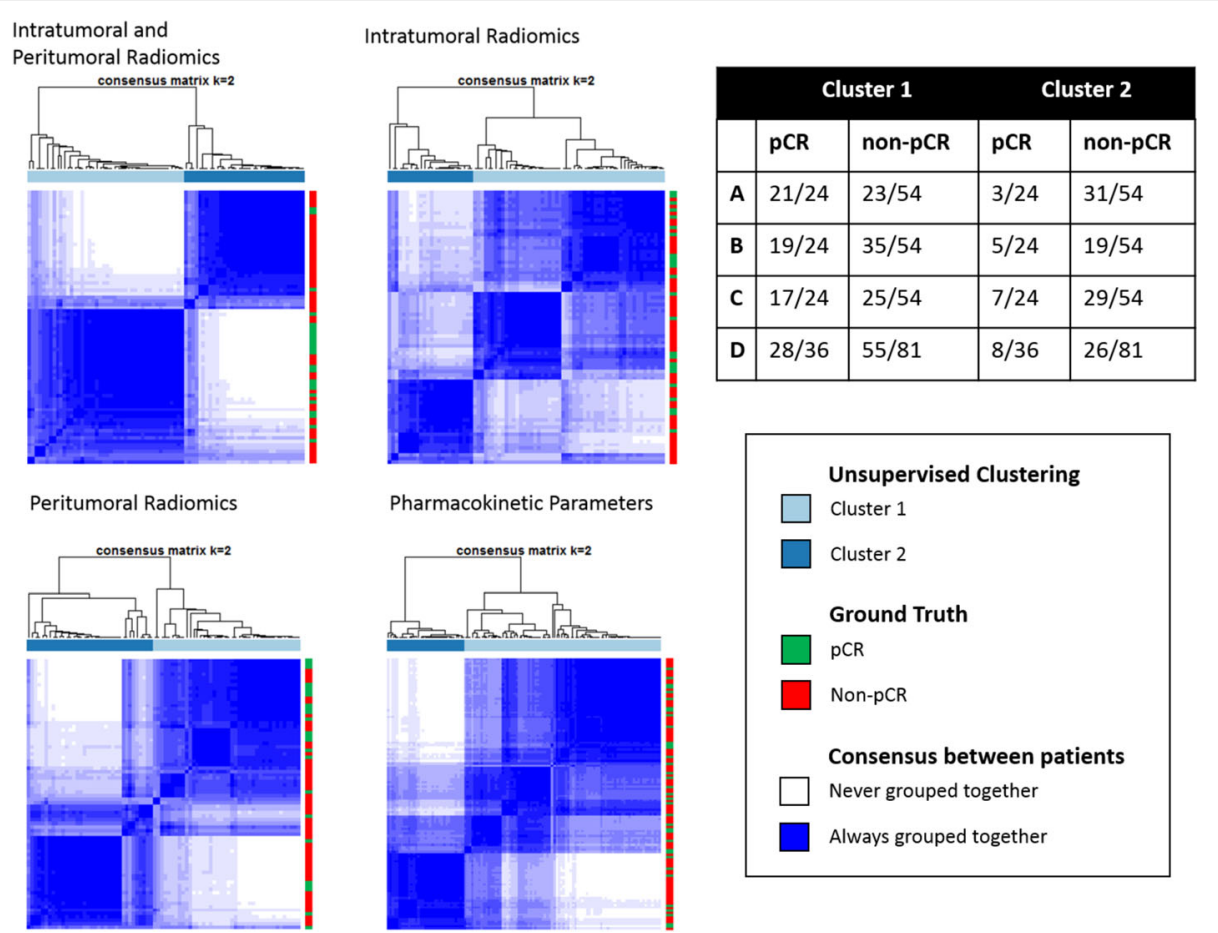

Fig. 2 Consensus clustering using combined peritumoral and intratumoral radiomics, intratumoral radiomics, peritumoral radiomics, and pharmacokinetic parameter feature sets. Combination of intratumoral and peritumoral features yielded clusters with the best consensus and correlation to pathological complete response $(p C R)$ status

$88 \%$ pCR and $57 \%$ non-pCR clustering accuracy. In Fig. $2 \mathrm{~b}$, consensus clustering using a set of the top ten intratumoral features produced groups with weak consensus and correlation with pCR, with cluster 1 receiving a majority of both pCR (79\%) and non-pCR (65\%) tumors. Clustering performed using only peritumoral features was less accurate than the combined peritumoral and intratumoral clustering (71\% pCR and 54\% non-pCR clustering accuracy) and had noticeably weaker consensus within clusters, as shown in Fig. 2c. PK parameters produced clusters with poor consensus and approximately proportional distribution of response groups (cluster 1 78\% pCR, 69\% non-pCR), as shown in Fig. 2d. Additionally, box plots of mean $K^{\text {Trans }}, K_{\mathrm{ep}}$, and $\mathrm{V}_{\mathrm{e}}$ (Additional file 2 ) showed no difference between pCR and non-pCR tumors for any of the experimental groups examined in this study.

Optimal classifier performance within the training set was achieved using a DLDA trained with 10 features across 50 threefold cross-validation iterations, yielding an average AUC of $0.78 \pm 0.03$ and accuracy of $0.76 \pm 0.03$. Prediction accuracy did not differ between patients using 1.5-T and 3$\mathrm{T}$ MRI scanners. Within the testing set, a DLDA classifier trained using only the top four features produced the strongest classification results. (AUC $=0.74$, accuracy $=0.67$ ). Of the 13 misclassified tumors, 7 were $\mathrm{HR}^{+}$, HER2 ${ }^{-}$nonresponders misidentified as pCR. The remaining six tumors were $\mathrm{TN} / \mathrm{HER} 2^{+}$(66\% true responders and 33\% true nonresponders). The classifier's accuracy within the testing set was greater for $\mathrm{HR}^{+}, \mathrm{HER} 2^{-}$tumors than for TN/HER2 ${ }^{+}$ tumors $(0.71$ vs. 0.60$)$. The 6 misclassified TN/HER $2^{+}$cases were comprised of 2 of the $4 \mathrm{TN}$ tumors (accuracy $=0.50$ ) and 4 of the $11 \mathrm{HER}^{+}$tumors (accuracy $=0.64$ ) within the testing set. The addition of subsequent features resulted in a decrease of both AUC and accuracy within the testing set. The top four radiomic features were successful in predicting pCR within the testing set regardless of classifier selection (Additional file 3: Tables S1-S5). Seven of thirteen misclassifications were imaged with a 3-T scanner.

\section{Experiment 2: pCR prediction with separation by receptor type}

\section{Feature discovery}

Half of the top ten $\mathrm{HR}^{+}, \mathrm{HER}^{-}$feature set obtained from feature discovery comprised CoLlAGe features. Laws, Gabor, and Haralick features were all represented within the top feature set as well. Six features were extracted from the peritumoral region and six from the initial post-contrast enhancement phase. The top three most discriminating features were all CoLlAGe entropyassociated descriptors expressed within the peritumoral region during the initial enhancement phase: kurtosis of entropy, skewness of difference entropy, and skewness of sum entropy. Top CoLlAGe feature expression indicates a more disordered peritumoral DCE-MRI phenotype 
among non-pCR $\mathrm{HR}^{+}$, HER2 ${ }^{-}$tumors, particularly during the initial phase of post-contrast enhancement. Figure $3 \mathrm{~b}$ shows qualitative feature maps of representative initial peritumoral CoLlAGe entropy for pCR and non-pCR breast cancers. In Fig. 3e, histology of a low CoLlAGe entropyexpressing $\mathrm{pCR}$ tumor shows brisk lymphocytic response at the tumor margins. Conversely, a non-pCR sample with high peritumoral entropy had significant tumor infiltration into surrounding adipose tissue. Additionally, intratumoral expression of low-frequency Gabor features was elevated during both initial and peak enhancement phases (features 6 and 8 ).

TN/HER2 ${ }^{+}$responder tumors were predominantly defined by Laws and CoLlAGe features, each comprising four of the ten top radiomic features. All top Laws features were computed peritumorally, with textural patterns of inconsistent enhancement such as waves, edges, and spots distinguishing $\mathrm{pCR}$ and non-pCR in both initial (spot-level, level-wave) and peak (level-ripple, edge-spot) enhancement phases. The top TN/HER2 ${ }^{+}$feature was the expression of Laws level-ripple during peak enhancement phase, shown qualitatively in Fig. 3c. Significant TIL presence was observed in a pCR sample with low Laws rippling, whereas high expression was found to correspond with a tumor with invasion of chondroid matrix into surrounding adipose tissue. Note that increased rippling in the peritumoral region of non-pCR tumors results in increased extreme positive and negative filter response values, whereas the more homogeneous $\mathrm{PCR}$ peritumoral appearance yields more consistent midrange values. CoLlAGe features quantifying homogeneity of orientation gradients-most notably initial intratumoral information measure of correlation 1, the top feature discovered in experiment 1 -comprised features 2,3 , and 4 . CoLlAGe

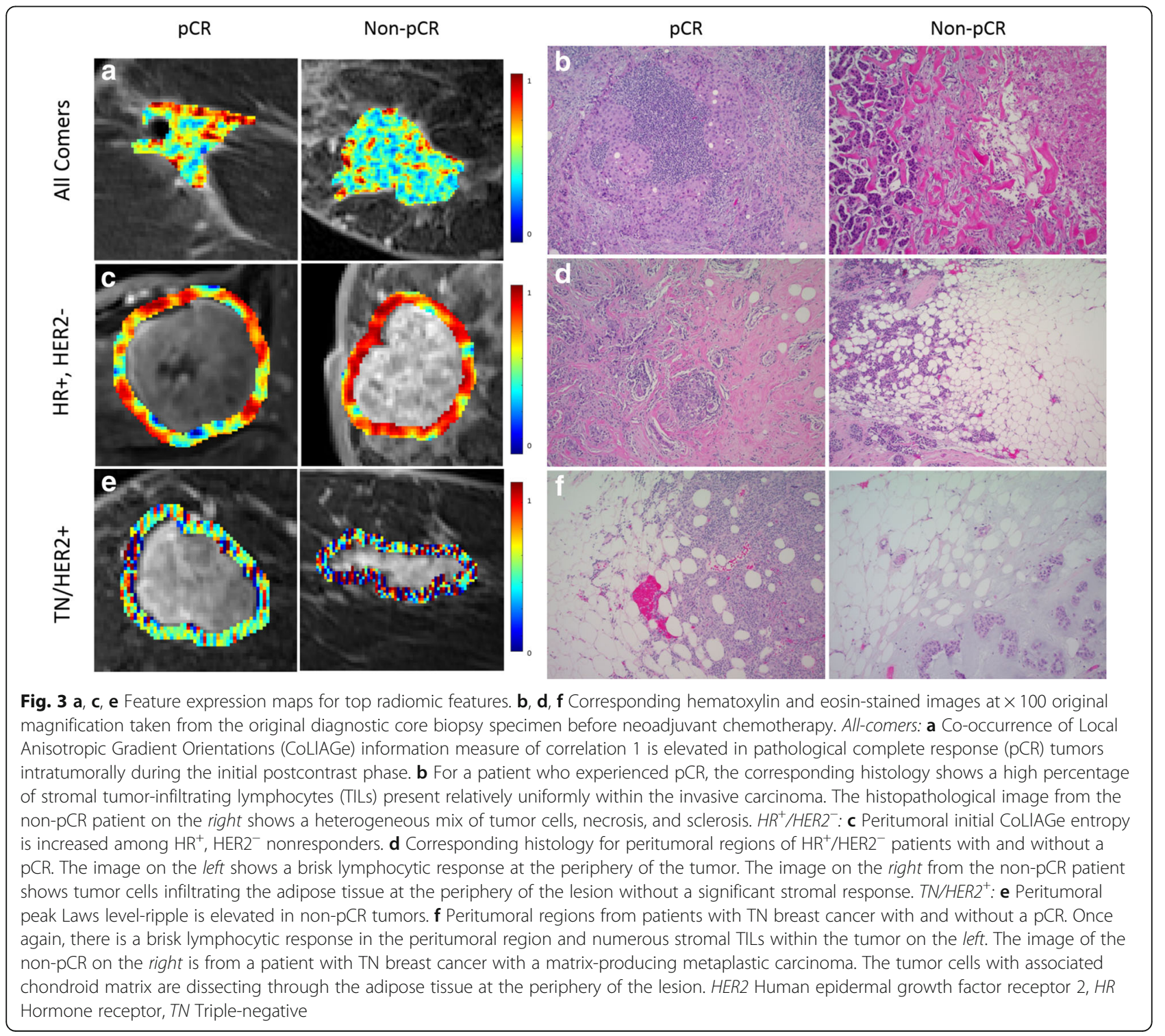


Table 4 Top ten radiomic features identified in each experiment

\begin{tabular}{|c|c|c|c|c|c|}
\hline Rank & Feature family & Descriptor & Location & Phase & Statistic \\
\hline \multicolumn{6}{|c|}{ Experiment 1} \\
\hline \multicolumn{6}{|c|}{ All-comers } \\
\hline 1 & CoLIAGe & Information measure of correlation 1 & Intratumoral & Initial & Kurtosis \\
\hline 2 & Laws & Spot-ripple & Intratumoral & Initial & Skewness \\
\hline 3 & Laws & Level-spot & Peritumoral & Peak & SD \\
\hline 4 & CoLIAGe & Entropy & Peritumoral & Initial & Kurtosis \\
\hline 5 & CoLIAGe & Sum average & Intratumoral & Peak & SD \\
\hline 6 & Laws & Edge-wave & Peritumoral & Initial & Mean \\
\hline 7 & CoLIAGe & Energy & Intratumoral & Initial & Median \\
\hline 8 & Laws & Wave-spot & Intratumoral & Initial & Skewness \\
\hline 9 & CoLIAGe & Inverse difference moment & Peritumoral & Peak & Kurtosis \\
\hline 10 & CoLIAGe & Correlation & Intratumoral & Peak & Kurtosis \\
\hline \multicolumn{6}{|c|}{ Experiment 2} \\
\hline \multicolumn{6}{|c|}{$\mathrm{HR}^{+}, \mathrm{HER} 2^{-}$} \\
\hline 1 & CoLIAGe & Entropy & Peritumoral & Initial & Kurtosis \\
\hline 2 & CoLIAGe & Difference entropy & Peritumoral & Initial & Skewness \\
\hline 3 & CoLIAGe & Sum entropy & Peritumoral & Initial & Skewness \\
\hline 4 & Laws & Level-ripple & Intratumoral & Peak & Kurtosis \\
\hline 5 & CoLIAGe & Inertia & Intratumoral & Peak & Skewness \\
\hline 6 & Gabor & $f=0, \theta=22.5$ degrees & Intratumoral & Initial & Median \\
\hline 7 & Laws & Edge-edge & Peritumoral & Initial & Skewness \\
\hline 8 & Gabor & $f=2, \theta=22.5$ degrees & Intratumoral & Initial & Kurtosis \\
\hline 9 & CoLIAGe & Inverse difference moment & Peritumoral & Peak & Skewness \\
\hline 10 & Haralick & Correlation & Peritumoral & Peak & SD \\
\hline \multicolumn{6}{|c|}{$\mathrm{TN} \mathrm{HER}^{+}$} \\
\hline 1 & Laws & Level-ripple & Peritumoral & Peak & Mean \\
\hline 2 & CoLIAGe & Information measure of correlation 1 & Intratumoral & Initial & Skewness \\
\hline 3 & CoLIAGe & Information measure of correlation 2 & Peritumoral & Peak & Mean \\
\hline 4 & CoLIAGe & Energy & Intratumoral & Initial & Median \\
\hline 5 & Laws & Spot-level & Peritumoral & Initial & Median \\
\hline 6 & Gabor & $f=2, \theta=135$ degrees & Intratumoral & Peak & Kurtosis \\
\hline 7 & Laws & Level-wave & Peritumoral & Initial & Median \\
\hline 8 & Laws & Edge-spot & Peritumoral & Peak & Skewness \\
\hline 9 & CoLIAGe & Difference variance & Peritumoral & Peak & Kurtosis \\
\hline 10 & Gabor & $f=2, \theta=22.5$ degrees & Intratumoral & Initial & Skewness \\
\hline
\end{tabular}

Abbreviations: CoLIAGe Co-occurrence of Local Anisotropic Gradient Orientations, HER2 Human epidermal growth factor receptor 2, HR Hormone receptor, TN Triple-negative

gradient orientation features were discriminative both intratumorally during initial phase (features 2 and 4 ) and peritumorally during peak enhancement (feature 3). Two lowfrequency Gabor features were also discriminative within the intratumoral region.

The top ten features identified within the all-comers, $\mathrm{HR}^{+} \mathrm{HER} 2^{-}$, and TN/HER2 ${ }^{+}$groups are included in Table 4. Note that the two highest-ranked features among all- comers were located within the intratumoral region; however, peritumoral features were ranked highest when feature discovery was performed within receptor typespecific groups.

\section{Validation}

Subtype-specific prediction vastly improved the ability to predict $\mathrm{pCR}$. Optimal response prediction was achieved 
with a ten-feature DLDA classifier, predicting pCR among $\mathrm{HR}^{+}, \mathrm{HER}^{-}$tumors with an AUC of $0.83 \pm 0.025$ and an accuracy of $0.79 \pm 0.033$. Classification sensitivity and specificity were $0.65 \pm 0.076$ and $0.82 \pm 0.038$, respectively. The ability of intratumoral and peritumoral radiomic features to predict $\mathrm{pCR}$ was shown to be robust across multiple classifiers (Additional file 3: Tables S1-S5).

A naive Bayes classifier trained with eight features was able to predict pCR with an average AUC of $0.93 \pm 0.018$ and accuracy of $0.84 \pm 0.030$. Sensitivity was $0.87 \pm 0.030$, and specificity was $0.81 \pm 0.047$. The accuracy of pCR prediction among $\mathrm{TN}$ tumors was $0.79 \pm 0.041$, whereas $\mathrm{HER}^{+}{ }^{+}$accuracy was $0.88 \pm 0.041$. The ability to predict $\mathrm{pCR}$ was consistent across all tested classifiers (Additional file 3: Tables S1-S5), including DLDA (AUC $=0.89 \pm 0.027$, accuracy $=0.83 \pm 0.030)$.

\section{Discussion}

In this study, we investigated the ability of radiomic textural features extracted from the intratumoral and peritumoral regions of pretreatment DCE-MRI to predict pCR to NAC. This approach was able to accurately distinguish between pCR and non-pCR in a highly heterogeneous cohort of pretreatment DCE-MRI scans. Most prior breast cancer radiomic approaches have been focused solely within the extent of the tumor itself [12-21, 24, 25] or the bulk parenchyma [22, 26-28]. The immediate surrounding tumor environment has remained relatively unexplored and may offer unique, orthogonal radiomic signatures of response prior to administration of NAC that enable enhanced $\mathrm{pCR}$ prediction.

In the feature discovery portion of this study, we attempted to define radiomic profiles of NAC responders. We found that CoLlAGe features measuring the consistency of local dominant gradient orientation differentiated pCR both intra- and peritumorally across all subtypes. Tumors that achieved $\mathrm{pCR}$ were characterized by increased expression of gradient homogeneity features within the tumor and decreased expression of gradient entropy features at the perimeter. Pretreatment diagnostic biopsy core samples for patients with representative radiomic expression (Fig. 3) revealed densely packed stromal TILs within the tumor that achieved pCR, likely contributing to a greater order of intratumoral intensity gradients as detected by CoLlAGe. This result is consistent with previous findings associating high TIL presence with more homogeneous DCE-MRI enhancement within the context of TN breast cancers [44]. The non-pCR tumor was found to contain intermingled tumor cells, necrosis, and sclerosis, likely contributing to the heterogeneity of the imaging appearance detected by CoLlAGe. These features might also be detecting enhancement heterogeneity due to increased chaotic angiogenesis within and surrounding tumors that do not achieve pCR; however, vascular marker staining of patient biopsies was not available to verify this hypothesis. Laws features capturing patterns of inconsistent enhancement such as speckling and rippling within the intratumoral and peritumoral regions were also found to discriminate between $\mathrm{pCR}$ and non-pCR.

We demonstrated that a combined intratumoral and peritumoral radiomic approach yielded clusters with high consensus and strong correlation with pCR ground truth in an unsupervised consensus clustering, outperforming radiomic analysis confined to a single region and physiologically based PK parameters. A DLDA classifier trained using the top four features identified during feature discovery predicted $\mathrm{pCR}$ with an AUC of 0.74 among the independent testing cohort. These features were successful in predicting pCR regardless of classifier chosen, suggesting that the features identified are robust predictors of response. These findings suggest that a combination of intratumoral and peritumoral cues may provide a more accurate and more comprehensive radiomic profile for characterization of breast cancers based on MRI.

Considering these results, we sought to determine whether the ability to predict response would improve with receptor status-specific feature sets and classifiers. In experiment 2, separating tumors by receptor status and repeating feature discovery improved the ability of an intratumoral and peritumoral radiomic classifier to predict $\mathrm{pCR}$. Among $\mathrm{HR}^{+}, \mathrm{HER} 2^{-}$tumors, a group strongly correlated with the luminal A molecular subtype [45], we could predict $\mathrm{pCR}$ with an $\mathrm{AUC}=0.83 \pm 0.025$. Owing to insufficient sample sizes of both TN and HER2 ${ }^{+}$patients, we grouped these patients into a combined TN/HER2 ${ }^{+}$ cohort. Relative to $\mathrm{HR}^{+}$, HER2 ${ }^{-}$breast cancers, these subtypes more frequently achieve pCR [46], and their response to NAC is more accurately detected on the basis of post-NAC MRI [47]. We demonstrated that separation of TN/HER2 ${ }^{+}$enabled more accurate prediction of $\mathrm{pCR}(\mathrm{AUC}=0.93 \pm 0.018)$ than in the $\mathrm{HR}^{+}$, HER2 ${ }^{-}$cohort, a surprising reversal of the receptor status group accuracies reported in experiment 1 .

Not only was pCR predicted more accurately using a receptor type-specific classifier but different biological groups also were found to possess unique radiomic profiles of response. $\mathrm{HR}^{+}$, HER2 ${ }^{-}$breast cancers were defined strongly by CoLlAGe features associated with entropy, whose expression was elevated in the peritumoral region of non-pCR tumors. An $\mathrm{HR}^{+}, \mathrm{HER} 2^{-}$patient with $\mathrm{pCR}$ and low peritumoral expression of CoLlAGe entropy features was observed to have a brisk lymphocytic response at the tumor's periphery. The non-pCR sample possessed significant infiltration of tumor cells into surrounding adipose tissue, potentially contributing to its high CoLlAGe-detected gradient entropy within the peritumoral region. These features could be detecting increasingly disordered enhancement gradients among 
nonresponders because of increased peritumoral vascularity and vascular invasion, an established marker of poor prognosis and risk of recurrence in $\mathrm{HR}^{+}, \mathrm{HER}^{-}$ breast cancers $[48,49]$. It is possible that peritumoral gradient orientation entropy during the initial enhancement phase was dominant within the top $\mathrm{HR}^{+}$, HER2 ${ }^{-}$ feature set because $\mathrm{HR}^{+}$, HER2 ${ }^{-}$tumors enhance more gradually than other subtypes $[50,51]$, making peritumoral vasculature abnormalities of nonresponders more apparent at the first postcontrast phase as compared with those who achieve pCR. Low-frequency wavelet features (Gabor) were also observed intratumorally, which have previously been found to detect architectural disorder of mammary glands on breast mammograms $[52,53]$.

In the TN/HER2 ${ }^{+}$cohort, Laws feature expression within the peritumoral region was found to be valuable in predicting $\mathrm{pCR}$. These features might be detecting textural patterns caused by surrounding stromal response or the presence of TILs at the tumor periphery, a predictor of pCR in both HER2 ${ }^{+}[54,55]$ and TN $[54,56]$ breast cancers, because Laws features have previously been shown to enable quantification of TILs in other imaging domains [57]. Examination of pretreatment biopsy samples substantiated this possibility, revealing brisk lymphocytic response and significant presence of TILs in a patient with pCR and low rippling in the peritumoral region as detected by Laws features. Conversely, the non-pCR patient observed to have the highest prevalence of rippling in the peritumoral region was discovered to have a matrix-producing metaplastic carcinoma with chondroid matrix infiltrating surrounding adipose tissue. Although not all TN/HER2 ${ }^{+}$non-pCR patients possess this phenotype, this finding does suggest that infiltration into the adipose tissue may manifest as a rippled pattern detectable using Laws features. CoLlAGe features within both the intratumoral and peritumoral regions, as well as low-frequency intratumoral Gabor features, also helped define the TN/HER2 ${ }^{+}$responder phenotype. TN and HER $2^{+}$breast cancers were also analyzed separately (detailed in Additional file 4: TN-specific and HER2 ${ }^{+}$-specific response prediction) to compare the identified radiomic response features for the individual molecular subgroups with those identified when the TN and HER2 ${ }^{+}$ cases were combined. We found that radiomic response features for the TN group to comprise Haralick, CoLlAGe, and Laws features from both the peritumoral and intratumoral regions, whereas the HER $2^{+}$response signature was composed almost entirely of Laws features from the peritumoral region during the peak enhancement phase (Additional file 4: Table S6). Response was predicted with average AUCs of 0.82 for TN and 0.80 for HER2 ${ }^{+}$, as compared with an AUC of 0.80 for combined TN/ $\mathrm{HER}^{+}$when using a comparable number of features and classifiers (Additional file 4: Table S7).
The work presented in this study did have its limitations, however. Although distinct training and testing cohorts were used in experiment 1 , we were unable to implement testing sets within the $\mathrm{HR}^{+}, \mathrm{HER}^{-}$, and TN/HER2 ${ }^{+}$subgroups, owing to insufficient $\mathrm{HR}^{+}, \mathrm{HER} 2^{-}$responders and total TN/HER2 ${ }^{+}$patients. AUCs reported for receptor status group-specific $\mathrm{pCR}$ prediction in experiment 2 were obtained by averaging cross-validation results within the same pool of patients used for the feature discovery phase (also performed using threefold cross-validation). As in experiment 1, these AUCs could decrease when evaluated in an independent testing cohort. However, receptor status group-specific AUCs still improved as compared with results of cross-validation within a training cohort of allcomers (AUC $=0.78 \pm 0.03$ ), suggesting that receptor status group-specific analysis would likely also enable stronger $\mathrm{pCR}$ prediction in an independent testing set. It is also possible that the elevated AUC within the TN/ HER $2^{+}$cohort could be partially due to a reduced sample size $(n=47)$ compared with the $\mathrm{HR}^{+}, \mathrm{HER}^{-}(n=$ $70)$ and all-comer training cohorts $(n=78)$; however, this result is consistent with previous findings that MRI has greater value in response prediction for TN/HER2 ${ }^{+}$ patients [47]. Our dataset also included diversity of treatment regimens within the all-comer and TN/HER2 ${ }^{+}$ groups, which might impact the likelihood of achieving pCR. In addition to the variability in prescribed treatment, the TN/HER2 ${ }^{+}$group featured two distinct receptor status groups. TN and HER2 ${ }^{+}$were combined into a single group, both on the basis of their correspondence to a nonluminal phenotype [45] and owing to the limited sample size. pCR was predicted in the TN/HER $2^{+}$group with the highest AUC of any of the patient groups analyzed, suggesting that these cancers seem to possess shared radiomic response signatures. However, these subtypes nonetheless represent distinct biologies and receive different treatment regimens. Grouping these tumors might possibly cause radiomic features that capture response signatures unique to HER2 ${ }^{+}$or TN breast cancers to be overlooked. For instance, radiomic features that detect HER2 enrichment, a predictor of improved response to HER2-targeted therapy [58], might strongly predict $\mathrm{pCR}$ in HER $2^{+}$tumors, but they would likely have little predictive value for TN breast cancer. As a result, these features might be missed during feature discovery in a combined TN/HER $2^{+}$dataset. Indeed, feature discovery within separated TN and HER2 ${ }^{+}$groups produced differing response signatures (Additional file 4: Table S6). In future work, we intend to explore TN-specific and $\mathrm{HER}^{+}{ }^{+}$-specific radiomic response signatures and their relationship with unique subtype biology with larger sample sizes. Additionally, our dataset is highly diverse with respect to the DCE-MRI protocol, with a multitude of scanners and heterogeneous magnet strengths. Although the rate of misclassification within the training set did not 
vary with magnet strength; 3-T scans were disproportionately misclassified in the testing set. Further investigation is required to determine the role that magnet strength may play in intratumoral and peritumoral radiomics. Owing to variance in voxel dimensions among scans, the dimensions of the peritumoral region occupies anywhere between a $2.5-$ to $5.0-\mathrm{cm}$ radius from the tumor. Further, the size of the peritumoral region varies widely on the basis of biological and clinical factors; therefore, approximating the peritumoral radius as a consistent distance from the tumor is an imperfect means of obtaining the peritumoral region. Analysis within recommended negative surgical margins was deemed a suitable stand-in for true peritumoral region borders that allowed quick sampling with minimum required clinical input. Future exploration of radiomic analysis within radiologist-annotated peritumoral regions may further improve the results presented in this study.

This work represents a preliminary success for the pretreatment prediction of $\mathrm{pCR}$ using intratumoral and peritumoral radiomics, which could potentially help guide personalized treatment of locally advanced breast cancers and reduce windows of ineffective treatment. Much work remains to be done, however, before its realization as a clinical tool. Although we were able to successfully demonstrate our ability to predict $\mathrm{pCR}$ among all-comers in experiment 1 with an independent holdout testing set, both these findings and the improved results of subtype-specific classifiers must next be validated within a larger independent dataset from possibly multiple external sites. Previous work [13] has suggested that radiomic features might be minimally affected by factors such as MRI magnetic strength. However, the Agner et al. study [13] was a limited study, and future work will entail a more detailed interrogation of the impact of scan parameters within the context of radiomic NAC response signatures. Further investigation may reveal the need for new standardization approaches to mitigate the effects of scan parameter heterogeneity. Additionally, greater intuition regarding the underlying biology captured by radiomic features will be a critical factor in their widespread adoption as clinical decision support tools. In this work, we conducted a qualitative assessment of representative patient histology in an attempt to unravel the morphometric and biological basis for the most predictive radiomic features presented. Future directions could include correlating radiomic features with both features from corresponding digital pathology surgical tissue sections and genomic measurements to more comprehensively characterize and identify the morphological and molecular basis of the most NAC-predictive radiomic features. Translation of this work will additionally require development of a platform with an intuitive user interface so that radiomic analysis could be easily implemented by doctors and integrated into current clinical workflows.

\section{Conclusions}

Patients with breast cancer who achieve pCR with NAC have a significantly improved prognosis and disease-free survival; however, only $10-50 \%$ of NAC recipients will achieve a full response [6]. There is a lack of imaging markers that enable noninvasive pretreatment prediction of pCR. In this study, we employed a novel combined intratumoral and peritumoral radiomic approach for $\mathrm{pCR}$ prediction, combining textural features extracted from a tumor and its immediate surrounding environment based on routine clinical DCE-MRI imaging. The efficacy of the approach was demonstrated in a relatively large and diverse imaging dataset. Specifically, we showed that peritumoral radiomics contribute to successful prediction of $\mathrm{pCR}$ from pretreatment imaging. Further, we identified molecular subtype-specific radiomic profiles of response and found that radiomic features most predictive of response appear to vary as a function of the molecular subtype of the tumor. These findings potentially hold significant clinical applications because they could help enable a clinical platform for the pretreatment prediction of $\mathrm{pCR}$ that would allow identification of patients most likely to achieve a response.

\section{Additional files}

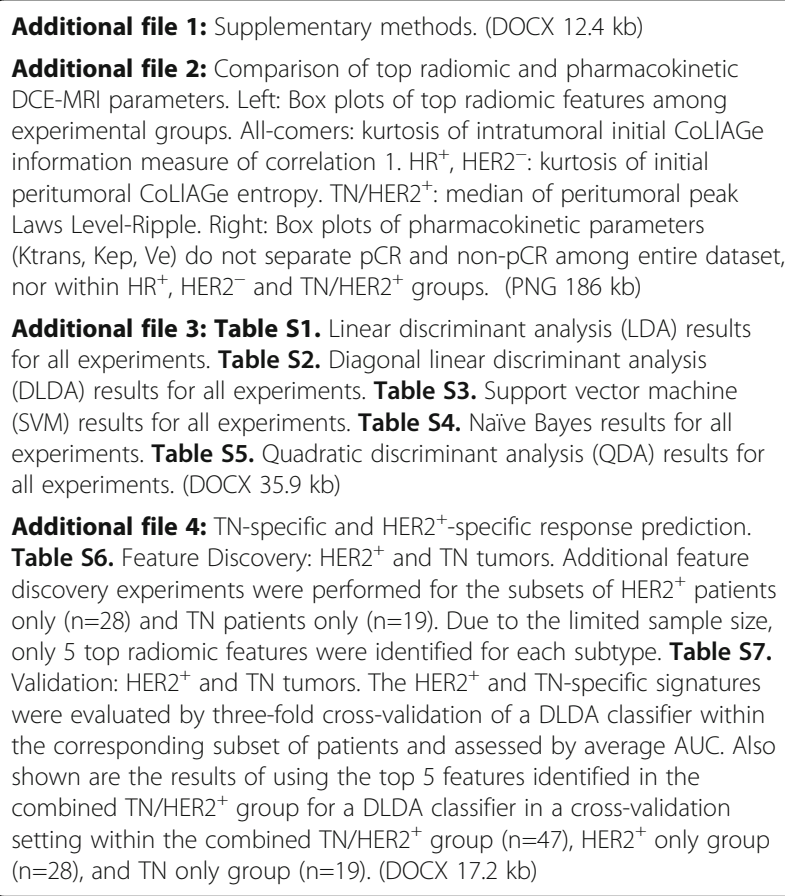

Additional file 3: Table S1. Linear discriminant analysis (LDA) results for all experiments. Table S2. Diagonal linear discriminant analysis (DLDA) results for all experiments. Table S3. Support vector machine (SVM) results for all experiments. Table S4. Naïve Bayes results for all experiments. Table S5. Quadratic discriminant analysis (QDA) results for all experiments. (DOCX $35.9 \mathrm{~kb}$ )

Additional file 4: $\mathrm{TN}$-specific and $\mathrm{HER} 2^{+}$-specific response prediction. Table S6. Feature Discovery: HER2 ${ }^{+}$and TN tumors. Additional feature discovery experiments were performed for the subsets of $\mathrm{HER}^{+}$patients only $(n=28)$ and TN patients only $(n=19)$. Due to the limited sample size, only 5 top radiomic features were identified for each subtype. Table S7. Validation: HER2 ${ }^{+}$and TN tumors. The HER2 ${ }^{+}$and TN-specific signatures were evaluated by three-fold cross-validation of a DLDA classifier within the corresponding subset of patients and assessed by average AUC. Also shown are the results of using the top 5 features identified in the combined TN/HER2 ${ }^{+}$group for a DLDA classifier in a cross-validation setting within the combined TN/HER2 ${ }^{+}$group $(n=47)$, HER2 ${ }^{+}$only group $(n=28)$, and TN only group $(n=19)$. (DOCX $17.2 \mathrm{~kb})$

\section{Abbreviations}

CoLIAGe: Co-occurrence of Local Anisotropic Gradient Orientations; CT: Computed tomography; DCE-MRI: Dynamic contrast-enhanced magnetic resonance imaging; DLDA: Diagonal linear discriminant analysis; ER: Estrogen receptor; HER2: Human epidermal growth factor receptor 2; HIPAA: Health Insurance Portability and Accountability Act of 1996; HR: Hormone receptor; LDA: Linear discriminant analysis; mRMR: Minimum redundancy, maximum relevancy; NAC: Neoadjuvant chemotherapy; pCR: Pathological complete 
response; PK: Pharmacokinetic; PR: Progesterone receptor; TIL: Tumor-infiltrating lymphocyte; TN: Triple-negative

\section{Acknowledgements}

The authors thank Elena DuPont for her support in the organization and transfer of patient data.

\section{Funding}

The research reported in this publication was supported by the National Cancer Institute of the National Institutes of Health under award numbers 1U24CA199374-01, R01 CA202752-01A1, R01 CA208236-01A1, R21 CA179327-01, and R21 CA195152-01; the National Institute of Biomedical Imaging and Bioengineering under award number T32EB007509; the National Institute of Diabetes and Digestive and Kidney Diseases under award number R01 DK098503-02; the National Center for Research Resources under award number 1 C06 RR12463-01; the U.S. Department of Defense (DOD) Prostate Cancer Synergistic Idea Development Award (PC120857); the DOD Lung Cancer Idea Development New Investigator Award (LC130463); the DOD Prostate Cancer Idea Development Award; DOD Peer Reviewed Medical Research Program W81XWH-16-1-0329; the Case Comprehensive Cancer Center Pilot Grant VelaSano Grant from the Cleveland Clinic; the Wallace H. Coulter Foundation Program in the Department of Biomedical Engineering at Case Western Reserve University; and the I-Corps@Ohio Program. The content is solely the responsibility of the authors and does not necessarily represent the official views of the National Institutes of Health.

\section{Availability of data and materials}

The datasets used and/or analyzed during the present study are available from the corresponding author on reasonable request.

\section{Authors' contributions}

$C D, D P, M E$, and $H G$ designed the clinical study from which patient data in this study derives and assisted with data collection. NMB and AM designed the retrospective radiomics study. ME and DP helped define the clinical problem, reviewed and annotated imaging data, and provided biological interpretation of the findings. NMB and PP performed processing of imaging data and feature extraction. NMB performed statistical analysis and drafted the manuscript and figures with input from $A M$. NMB, AM, ME, PP, HG, DP, and PT were involved with the interpretation of the findings. PP, AM, and PT provided guidance on the implementation and interpretation of CoLIAGe. HG provided guidance regarding peritumoral biology and examined histology of characteristic patient samples. All authors contributed to editing the manuscript and read and approved the final version.

\section{Competing interests}

AM is the cofounder and stakeholder in Ibris Inc., a cancer diagnostics company. Additionally, AM is an equity holder in Elucid Bioimaging and in Inspirata Inc. AM is also a scientific advisory consultant for Inspirata Inc. DP is a consultant for Hologic, Inc.

\section{Consent for publication}

Not applicable.

\section{Ethics approval and consent to participate}

The study was compliant with Health Insurance Portability and Accountability Act of 1996 ("HIPAA") regulations and was approved by the institutional review board at the University Hospitals Case Medical Center. The need for informed consent was waived by the institutional review board for this retrospective study.

\section{Publisher's Note}

Springer Nature remains neutral with regard to jurisdictional claims in published maps and institutional affiliations.

\section{Author details}

'Department of Biomedical Engineering, Case Western Reserve University, Cleveland, OH 44106, USA. ${ }^{2}$ University Hospitals Case Medical Center, Cleveland, OH 44106, USA.
Received: 29 December 2016 Accepted: 25 April 2017 Published online: 18 May 2017

\section{References}

1. American Cancer Society. Breast cancer facts \& figures. http://www.cancer.org/ research/cancerfactsstatistics/breast-cancer-facts-figures. Accessed 8 Dec 2016.

2. Giordano SH. Update on locally advanced breast cancer. Oncologist. 2003;8:521-30.

3. Thompson AM, Moulder-Thompson SL. Neoadjuvant treatment of breast cancer. Ann Oncol. 2012;23 Suppl 10:x231-6.

4. Luangdilok S, Samarnthai N, Korphaisarn K. Association between pathological complete response and outcome following neoadjuvant chemotherapy in locally advanced breast cancer patients. J Breast Cancer. 2014;17:376-85.

5. Kong X, Moran MS, Zhang N, Haffty B, Yang Q. Meta-analysis confirms achieving pathological complete response after neoadjuvant chemotherapy predicts favourable prognosis for breast cancer patients. Eur J Cancer. 2011;47:2084-90.

6. Earl H, Provenzano E, Abraham J, Dunn J, Vallier AL, Gounaris I, et al. Neoadjuvant trials in early breast cancer: pathological response at surgery and correlation to longer term outcomes - what does it all mean? BMC Med. 2015;13:234.

7. Marinovich ML, Sardanelli F, Ciatto S, Mamounas E, Brennan M, Macaskill P, et al. Early prediction of pathologic response to neoadjuvant therapy in breast cancer: systematic review of the accuracy of MRI. Breast. 2012;21:669-77.

8. Delille JP, Slanetz PJ, Yeh ED, Halpern EF, Kopans DB, Garrido L. Invasive ductal breast carcinoma response to neoadjuvant chemotherapy: noninvasive monitoring with functional MR imaging pilot study. Radiology. 2003:228:63-9.

9. Padhani AR, Hayes C, Assersohn L, Powles T, Makris A, Suckling J, et al. Prediction of clinicopathologic response of breast cancer to primary chemotherapy at contrast-enhanced MR imaging: initial clinical results. Radiology. 2006;239:361-74.

10. Dialani V, Chadashvili T, Slanetz PJ. Role of imaging in neoadjuvant therapy for breast cancer. Ann Surg Oncol. 2015;22:1416-24.

11. Yip SSF, Aerts HJWL. Applications and limitations of radiomics. Phys Med Biol. 2016;61:R150-66.

12. Zhu Y, Li H, Guo W, Drukker K, Lan L, Giger ML, et al. Deciphering genomic underpinnings of quantitative MRI-based radiomic phenotypes of invasive breast carcinoma. Sci Rep. 2015;5:17787.

13. Agner SC, Rosen MA, Englander S, Tomaszewski JE, Feldman MD, Zhang P, et al. Computerized image analysis for identifying triple-negative breast cancers and differentiating them from other molecular subtypes of breast cancer on dynamic contrast-enhanced MR images: a feasibility study. Radiology. 2014;272:91-9.

14. Ashraf A, Gaonkar B, Mies C, DeMichele A, Rosen M, Davatzikos C, et al. Breast DCE-MRI kinetic heterogeneity tumor markers: preliminary associations with neoadjuvant chemotherapy response. Transl Oncol. 2015;8:154-62.

15. Guo W, Li H, Zhu Y, Lan L, Yang S, Drukker K, et al. Prediction of clinical phenotypes in invasive breast carcinomas from the integration of radiomics and genomics data. J Med Imaging (Bellingham). 2015;2:041007.

16. Burnside ES, Drukker K, Li H, Bonaccio E, Zuley M, Ganott M, et al. Using computerextracted image phenotypes from tumors on breast magnetic resonance imaging to predict breast cancer pathologic stage. Cancer. 2016;122:748-57.

17. Li H, Lan L, Drukker K, Perou C, Giger M. TU-AB-BRA-08: radiomics in the analysis of breast cancer heterogeneity on DCE-MRI [abstract]. Med Phys. 2015;42:3588.

18. Kim JH, Ko ES, Lim Y, Lee KS, Han BK, Ko EY, et al. Breast cancer heterogeneity: MR imaging texture analysis and survival outcomes. Radiology. 2017;282:665-75.

19. Wu J, Gong G, Cui Y, Li R. Intratumor partitioning and texture analysis of dynamic contrast-enhanced (DCE)-MRI identifies relevant tumor subregions to predict pathological response of breast cancer to neoadjuvant chemotherapy. J Magn Reson Imaging. 2016:44:1107-15.

20. Li H, Zhu Y, Burnside ES, Huang E, Drukker K, Hoadley KA, et al. Quantitative MRI radiomics in the prediction of molecular classifications of breast cancer subtypes in the TCGA/TCIA data set. NPJ Breast Cancer. 2016;2:16012.

21. Waugh SA, Purdie CA, Jordan LB, Vinnicombe S, Lerski RA, Martin P, et al. Magnetic resonance imaging texture analysis classification of primary breast cancer. Eur Radiol. 2016;26:322-30.

22. Wang J, Kato F, Oyama-Manabe N, Li R, Cui Y, Tha KK, et al. Identifying triple-negative breast cancer using background parenchymal enhancement heterogeneity on dynamic contrast-enhanced MRI: a pilot radiomics study. PLoS One. 2015;10:e0143308. 
23. Sutton EJ, Dashevsky BZ, Oh JH, Veeraraghavan H, Apte AP, Thakur SB, et al. Breast cancer molecular subtype classifier that incorporates MRI features. J Magn Reson Imaging. 2016;44:122-9.

24. Wan T, Bloch BN, Plecha D, Thompson CL, Gilmore H, Jaffe C, et al. A radiogenomics approach for identifying high risk estrogen receptor-positive breast cancers on DCE-MRI: preliminary results in predicting OncotypeDX risk scores. Sci Rep. 2016;6:21394.

25. Li H, Zhu Y, Burnside ES, Drukker K, Hoadley KA, Fan C, et al. MR imaging radiomics signatures for predicting the risk of breast cancer recurrence as given by research versions of MammaPrint, Oncotype DX, and PAM50 gene assays. Radiology. 2016;281:382-91.

26. Keller BM, Chen J, Conant EF, Kontos D. Breast density and parenchymal texture measures as potential risk factors for estrogen-receptor positive breast cancer. Proc SPIE. 2014;9035:90351D.

27. Wu S, Weinstein SP, DeLeo 3rd MJ, Conant EF, Chen J, Domchek SM, et al, Quantitative assessment of background parenchymal enhancement in breast MRI predicts response to risk-reducing salpingo-oophorectomy: preliminary evaluation in a cohort of BRCA1/2 mutation carriers. Breast Cancer Res. 2015;17:67.

28. Wu S, Berg WA, Zuley ML, Kurland BF, Jankowitz RC, Nishikawa R, et al. Breast MRI contrast enhancement kinetics of normal parenchyma correlate with presence of breast cancer. Breast Cancer Res. 2016;18:76

29. Locopo N, Fanelli M, Gasparini G. Clinical significance of angiogenic factors in breast cancer. Breast Cancer Res Treat. 1998;52:159-73.

30. Christiansen A, Detmar M. Lymphangiogenesis and cancer. Genes Cancer. 2011;2: 1146-58.

31. Mohammed ZM, McMillan DC, Edwards J, Mallon E, Doughty JC, Orange $C$, et al. The relationship between lymphovascular invasion and angiogenesis, hormone receptors, cell proliferation and survival in patients with primary operable invasive ductal breast cancer. BMC Clin Pathol. 2013;13:31.

32. Lee AK, DeLellis RA, Silverman ML, Heatley GJ, Wolfe HJ. Prognostic significance of peritumoral lymphatic and blood vessel invasion in nodenegative carcinoma of the breast. J Clin Oncol. 1990;8:1457-65.

33. Conklin MW, Keely PJ. Why the stroma matters in breast cancer. Cell Adhes Migr. 2012;6:249-60.

34. Ocaña A, Diez-Gónzález L, Adrover E, Fernández-Aramburo A, Pandiella A, Amir $E$. Tumor-infiltrating lymphocytes in breast cancer: ready for prime time? J Clin Oncol. 2015;33:1298-9.

35. Tofts PS. Modeling tracer kinetics in dynamic Gd-DTPA MR imaging. J Magn Reson Imaging. 1997:7:91-101.

36. Ding $\mathrm{C}$, Peng $\mathrm{H}$. Minimum redundancy feature selection from microarray gene expression data. J Bioinform Comput Biol. 2005;3:185-205.

37. Foley D. Considerations of sample and feature size. IEEE Trans Inf Theory. 1972;18:618-26.

38. Brown G, Pocock A, Zhao MJ, Luján M. Conditional likelihood maximisation: a unifying framework for information theoretic feature selection. J Mach Learn Res. 2012;13:27-66.

39. Monti S, Tamayo P, Mesirov JP, Golub TR. Consensus clustering: a resampling-based method for class discovery and visualization of gene expression microarray data. Mach Learn. 2003;52:91-118.

40. Wilkerson MD, Hayes DN. ConsensusClusterPlus: a class discovery tool with confidence assessments and item tracking. Bioinformatics. 2010;26:1572-3.

41. R Core Team. R: A language and environment for statistical computing [Internet]. 2015. Vienna: R Foundation for Statistical Computing. https://www.R-project.org/

42. Parmar C, Leijenaar RTH, Grossmann P, Rios Velazquez E, Bussink J, Rietveld $D$, et al. Radiomic feature clusters and prognostic signatures specific for lung and head \& neck cancer. Sci Rep. 2015;5:11044.

43. Ressom HW, Varghese RS, Zhang Z, Xuan J, Clarke R. Classification algorithms for phenotype prediction in genomics and proteomics. Front Biosci J Virtual Libr. 2008;13:691-708.

44. Ku YJ, Kim HH, Cha JH, Shin HJ, Baek SH, Lee HJ, et al. Correlation between $\mathrm{MRI}$ and the level of tumor-infiltrating lymphocytes in patients with triplenegative breast cancer. AJR Am J Roentgenol. 2016;207:1146-51.

45. Onitilo AA, Engel JM, Greenlee RT, Mukesh BN. Breast cancer subtypes based on ER/PR and Her2 expression: comparison of clinicopathologic features and survival. Clin Med Res. 2009;7:4-13.

46. Houssami N, Macaskill P, von Minckwitz G, Marinovich ML, Mamounas E. Metaanalysis of the association of breast cancer subtype and pathologic complete response to neoadjuvant chemotherapy. Eur J Cancer. 2012;48:3342-54.
47. Price ER, Wong J, Mukhtar R, Hylton N, Esserman LJ. How to use magnetic resonance imaging following neoadjuvant chemotherapy in locally advanced breast cancer. World J Clin Cases. 2015;3:607-13.

48. Jacquemier J, Charafe-Jauffret E, Monville F, Esterni B, Extra JM, Houvenaeghel G, et al. Association of GATA3, P53, Ki67 status and vascular peritumoral invasion are strongly prognostic in luminal breast cancer. Breast Cancer Res. 2009;11:R23.

49. Munzone E, Bagnardi V, Rotmensz N, Sporchia A, Mazza M, Pruneri G, et al. Prognostic relevance of peritumoral vascular invasion in immunohistochemically defined subtypes of node-positive breast cancer. Breast Cancer Res Treat. 2014;146:573-82.

50. Blaschke $E, A b e ~ H . M R I$ phenotype of breast cancer: kinetic assessment for molecular subtypes. J Magn Reson Imaging. 2015:42:920-4.

51. Kawashima H, Inokuchi M, Furukawa H, Ikeda H, Kitamura S. Magnetic resonance imaging features of breast cancer according to intrinsic subtypes: correlations with neoadjuvant chemotherapy effects. Springerplus. 2014;3:240.

52. Yoshikawa R, Teramoto A, Matsubara T, Fujita H. Automated detection of architectural distortion using improved adaptive Gabor filter. In: Fujita H, Hara T, Muramatsu C, editors. Breast Imaging. IWDM. Lecture Notes in Computer Science. 2014;8539:606-11.

53. Rangayyan RM, Banik S, Chakraborty J, Mukhopadhyay S, Desautels JEL. Measures of divergence of oriented patterns for the detection of architectural distortion in prior mammograms. Int J Comput Assist Radiol Surg. 2012:8:527-45.

54. Dushyanthen S, Beavis PA, Savas P, Teo ZL, Zhou C, Mansour M, et al. Relevance of tumor-infiltrating lymphocytes in breast cancer. BMC Med. 2015;13:202.

55. Liu S, Duan X, Xu L, Xin L, Cheng Y, Liu Q, et al. Optimal threshold for stromal tumor-infiltrating lymphocytes: its predictive and prognostic value in HER2-positive breast cancer treated with trastuzumab-based neoadjuvant chemotherapy. Breast Cancer Res Treat. 2015;154:239-49.

56. Ono M, Tsuda H, Shimizu C, Yamamoto S, Shibata T, Yamamoto H, et al. Tumorinfiltrating lymphocytes are correlated with response to neoadjuvant chemotherapy in triple-negative breast cancer. Breast Cancer Res Treat. 2012;132:793-805.

57. Orooji M, Rakshit S, Beig N, Madabhushi A, Velcheti V. Computerized textural analysis of lung $\mathrm{CT}$ to enable quantification of tumor infiltrating lymphocytes in NSCLC [abstract]. J Clin Oncol. 2016:34:11584.

58. Prat A, Pineda E, Adamo B, Galván P, Fernández A, Gaba L, et al. Clinical implications of the intrinsic molecular subtypes of breast cancer. Breast. 2015;24 Suppl 2:S26-35.

59. Prasanna P, Tiwari P, Madabhushi A. Co-occurrence of Local Anisotropic Gradient Orientations (CoLIAGe): distinguishing tumor confounders and molecular subtypes on MRI. Med Image Comput Comput Assist Interv. 2014;17:73-80.

60. Prasanna P, Tiwari P, Madabhushi A. Co-occurrence of Local Anisotropic Gradient Orientations (CoLIAGe): a new radiomics descriptor. Sci Rep. 2016:6:37241.

\section{Submit your next manuscript to BioMed Central and we will help you at every step:}

- We accept pre-submission inquiries

- Our selector tool helps you to find the most relevant journal

- We provide round the clock customer support

- Convenient online submission

- Thorough peer review

- Inclusion in PubMed and all major indexing services

- Maximum visibility for your research

Submit your manuscript at www.biomedcentral.com/submit 